\title{
AN ELEMENTARY PROOF OF A FUNDAMENTAL LEMMA CONCERNING THE LIMIT OF A SUM*
}

\author{
BY H. J. ETTLINGER
}

It is the object of this note to give a simple proof of the following lemma upon which may be based the theory of the Riemann integral.

Let the interval $I: a \leqq x \leqq b$ be divided into $n$ equal subintervals, $I(i, n)$, each of length $\Delta_{n} x=(b-a) / n$. To each $I(i, n)$ there corresponds a number $h(i, n)$. Let $|h(i, n)| \leqq M$ for all values of $i(\leqq n)$ and $n$, where $M$ is a constant. If $x$ is any fixed value in $I$, then for each value of $n, x$ is contained in at least one of the sub-intervals. Let it be designated by $I(x, n)$. For each fixed $x$ and each subdivision $I(x, n)$ let the corresponding number be $h(x, n)$, and let $\lim _{n \rightarrow \infty} h(x, n)=0$. Then

$$
\lim _{n \rightarrow \infty} \sum_{1}^{n} h(i, n) \Delta_{n} x=0 .
$$

In a recent paper $\dagger$ the above lemma was stated in terms of area as a geometric theorem and used to develop the theorems on definite integrals of fundamental importance in the integral calculus. In addition, applications were given to the transformation of a double integral by change of variables and to the Fredholm method of solution of an integral equation.

A proof based on a generalization of a theorem due to $\mathrm{W}$. H. Young $\ddagger$ has been given by R. L. Moore. $\S$ For the proof of the above lemma, however, it is sufficient to use Arzelà's \| "lemma fondamentale" which may be stated in terms of the

* Presented to the Society, September 7, 1922.

$\dagger A$ simple form of Duhamel's theorem and some new applications, American Mathematical Monthly, vol. 29 (1922), p. 239.

$\ddagger$ Open sets and the theory of content, Procendings of the London SocIETY, (2), vol. 2 (1904), pp. 20-22.

$\S$ On Duhamel's theorem, Annals of Mathematics, (2), vol. 13 (1912), p. 163.

\|Un teorema intorno alle serie di funzioni, Lincei Rendiconti, (4), vol. 1 (1885), pp. 262-267. 
notation of the above theorem as follows:

If for each $n, I(n)=\sum_{1}^{k} I\left(m_{i}, n\right)$, where $m_{i}$ is a positive integer such that

$$
1 \leqq m_{1}<m_{2}<\cdots<m_{k} \leqq n,
$$

and $I \geqq I(n)>\delta>0$ for every $n$, there is at least one point $P$ which is common to an infinite number of the $I(n)$ 's.

The proof of the lemma may also be based on the following theorem of Osgood.*

If $s_{n}(x)$ is continuous in $a \leqq x \leqq b$ and converges when $n \rightarrow \infty$ for every $x$ in the interval, and if $\left|s_{n}(x)\right| \leqq M$ for all values of $n=1,2, \cdots$ and $x$ in $a \leqq x \leqq b$, where $M$ is $a$ constant, then

$$
\lim _{n \rightarrow \infty} \int_{a}^{b} s_{n}(x) d x=\int_{a}^{b} \lim _{n \rightarrow \infty} s_{n}(x) d x .
$$

This theorem is applied as follows. On $I(i, n)$ as base erect an isosceles triangle whose altitude is $2 h(i, n)$. The equal legs of these triangles form the graph of $s_{n}(x)$. Identify

$$
\sum_{1}^{h} h(i, n) \Delta_{n} x=\int_{a}^{b} s_{n}(x) d x .
$$

Finally note that $\lim _{n \rightarrow \infty} s_{n}(x)=0$ for every fixed value of $x$, and the lemma is proved.

Other proofs of Osgood's theorem have been given by F. Riesz, $\dagger$ Bieberbach, $\ddagger$ and Landau. $\S$ The method given by Landau is substantially that given by Moore (loc. cit.) and makes use of Arzelà's "lemma fondamentale" (loc. cit.). The proof by Bieberbach is essentially the same as that of Landau, with the exception that a simplified proof of Arzelà's theorem is included. The method of Riesz is more elementary and has suggested the proof given in this note.

It is to be noticed mutatis mutandis that Osgood's theorem

* On non-uniform convergence and integration of a series term by term, American Journal, vol. 19 (1897), p. 188.

$\dagger$ Ueber Integration unendlicher Folgen, JAHRESBerICHT DER VereINIGUNG, vol. 26 (1917-18), pp. 274-278.

$\ddagger$ Ueber einen Osgoodschen Satz aus der Integralrechnung, MathemaTISCHE ZEITSCHRIFT, vol. 2 (1918), pp. 156-157.

§ Ein Satz ueber Riemannsche Integrale, Mathematische Zeitschrift, vol. 2 (1918), pp. 350-351. 
may be regarded as a corollary of the lemma of this note. It would seem to be more natural to establish the lemma stated above by elementary methods and derive from it the properties of the Riemann integral, of which the theorem of Osgood would be one.

We proceed to the proof. Let

Then

$$
f(n)=\sum_{1}^{n}|h(i, n)| \Delta_{n} x .
$$

$$
f(n) \geqq\left|\sum_{1}^{n} h(i, n) \Delta_{n} x\right| \geqq 0 .
$$

If $\lim _{n \rightarrow \infty} f(n)=0$ is proved, the lemma is established.

CASE 1. Let $|h(x, n+1)| \leqq|h(x, n)|$ for every fixed $x$ in I. Then $f(n) \geqq f(n+1) \geqq 0$, or $f(n)$ is a monotonically nonincreasing function of $n$. Hence

$$
\lim _{n \rightarrow \infty} f(n)=A \geqq 0 .
$$

Divide $I$ into two equal parts. Then $\left|h\left(i_{2}, 2\right)\right| \Delta_{2} x \geqq A / 2$, where $i_{2}$ has at least one of the two values, $i=1,2$. Divide $I\left(i_{2}, 2\right)$ into two equal parts. Then $\left|h\left(i_{4}, 4\right)\right| \Delta_{4} x \geqq A / 2^{2}$, where $i_{4}$ has at least one value of the set $1,2,3,4$. If this process be continued $k$ times, we have $\left|h\left(i_{m}, m\right)\right| \Delta_{m} x \geqq A / m$, where $m=2^{k}$ and $i_{m}$ has at least one value of the set 1,2 , $\cdots, 2^{k}$. But $\Delta_{m} x=(b-a) / m$. Hence $A \leqq\left|h\left(i_{m}, m\right)\right|(b-a)$.

The closed sub-intervals $I\left(i_{2}, 2\right), I\left(i_{4}, 4\right), \cdots, I\left(i_{m}, m\right)$ are so related that each is contained in the preceding, and, as $n$ increases without limit, $\Delta_{m} x$ approaches zero. Therefore there exists a point $\bar{P}$ of $I$ whose abscissa is $\bar{x}$ such that $\bar{P}$ is common to all these sub-intervals. Hence $h\left(i_{2}, 2\right), h\left(i_{4}, 4\right)$, ... form a set of numbers $h(\bar{x}, n)$ corresponding to $\bar{x}$. But $\lim _{n \rightarrow \infty}|h(\bar{x}, n)|=0$. Hence $A=0$. This same method with a slight modification can be used for this case if the length of the subdivisions are unequal.

CASE 2. For the general case, let $h(x, n, m)$ for $n \leqq m$ be the greatest of the numbers $|h(x, n)|,|h(x, n+1)|, \cdots$, $|h(x, m)|$. Form

$$
\phi(n, m)=\sum_{1}^{n} h(i, n, m) \Delta_{n} x
$$


where $h(i, n, m) \Delta_{n} x$ is the area of the rectangles whose bases fill up $I(i, n)$ and whose heights $h(i, n, m)$ are chosen so as to satisfy the preceding condition for each $x$ in $I(i, n)$. Now $h(x, n, m) \leqq M$ for all values of $m$ and $n \leqq m$. Hence

$$
\phi(n, m) \leqq M(b-a) .
$$

For fixed $x$ and $n, h(x, n, m) \leqq h(x, n, m+1)$. Hence

$$
\phi(n, m) \leqq \phi(n, m+1) \text {. }
$$

So that for a fixed $n, \phi(n, m)$ is a monotonically nondecreasing function of $m$. Hence $\lim _{m \rightarrow \infty} \phi(n, m)=H(n)$ $\leqq M(b-a)$, and

$$
\phi(n, m) \leqq H(n)
$$

But $f(n) \leqq \phi(n, m)$, or $f(n) \leqq H(n)$. Hence $\lim _{n \rightarrow \infty} H(n)=0$ will carry with it $\lim _{n \rightarrow \infty} f(n)=0$.

Given $\delta>0$ and arbitrarily small, define a sequence of positive integers, $m_{i}$, to satisfy the inequality

$$
\phi\left(i, m_{i}\right) \geqq H(i)-\frac{\delta}{2^{i}}, \quad(i=1,2, \cdots, n) .
$$

Since $\phi(i, m) \geqq H(i)-\left(\delta / 2^{i}\right)$ for $m>m_{i}$ we may choose the set $m_{i}$ to satisfy $m_{1}<m_{2}<m_{3}<\cdots<m_{n}<\cdots$. Let $\bar{h}(x, n)$ denote the smallest of the numbers $h\left(x, 1, m_{1}\right)$, $h\left(x, 2, m_{2}\right), \cdots, h\left(x, n, m_{n}\right)$. Form

$$
\psi(n)=\sum_{1}^{n} \bar{h}(i, n) \Delta_{n} x,
$$

where, as above, $\bar{h}(i, n) \Delta_{n} x$ is the area of the rectangles whose bases fill up $I(i, n)$ and whose heights $\bar{h}(i, n)$ are chosen so as to satisfy the preceding condition for each $x$ in $I(i, n)$.

We shall prove by induction that

$$
\psi(n)>H(n)-\delta\left(1-\frac{1}{2^{n}}\right) .
$$

For $n=1$ we have $\bar{h}(x, 1) \equiv h(x, 1, m)$, and hence $\phi\left(1, m_{1}\right)$ $\equiv \psi(1)$. Hence by (2), (3) is true for $n=1$. If (3) is not true for every positive integer, let $k$ be the first integer for which it is not true. Then (3) is true for $n=k-1$, or

$$
\psi(k-1)>H(k-1)-\delta\left(1-\frac{1}{2^{k-1}}\right) .
$$

Now

(5) $\bar{h}(x, k) \leqq \bar{h}(x, k-1)$, and $\bar{h}(x, k) \leqq h\left(x, k, m_{k}\right)$. 
But

(6) $\bar{h}(x, k-1) \leqq h\left(x, k-1, m_{k-1}\right) \leqq h\left(x, k-1, m_{k}\right)$.

Compare

and

$$
\vec{h}(x, k-1)-\bar{h}(x, k) \geqq 0
$$

$$
h\left(x, k-1, m_{k}\right)-h\left(x, k, m_{k}\right) \geqq 0 .
$$

If $\bar{h}(x, k-1) \leqq h\left(x, k, m_{k}\right)$, then $\bar{h}(x, k-1)-\bar{h}(x, k)=0$, and hence in this case

$$
\bar{h}(x, k-1)-\bar{h}(x, k) \leqq h\left(x, k-1, m_{k}\right)-h\left(x, k, m_{k}\right) .
$$

If $\vec{h}(x, k-1)>h\left(x, k, m_{k}\right)$, then

$$
\vec{h}(x, k)=h\left(x, k, m_{k}\right) \text {. }
$$

Subtracting (7) from (6), we have

$$
\bar{h}(x, k-1)-\bar{h}(x, k) \leqq h\left(x, k-1, m_{k}\right)-h\left(x, k, m_{k}\right) .
$$

Hence in every case

(8) $\vec{h}(x, k) \geqq \bar{h}(x, k-1)+h\left(x, k, m_{k}\right)-h\left(x, k-1, m_{k}\right)$, for every $x$ in $I$. Then

$$
\psi(k) \geqq \psi(k-1)+\phi\left(k, m_{k}\right)-\phi\left(k-1, m_{k}\right) .
$$

By (4), (2), and (1) we may strengthen (9):

$\psi(k) \geqq H(k-1)-\delta\left(1-\frac{1}{2^{k-1}}\right)+H(k)-\frac{\delta}{2 k}-H(k-1)$, or

$$
\psi(k) \geqq H(k)-\delta\left(1-\frac{1}{2 k}\right) \cdot
$$

This proves (3). Now strengthen (3) and write

But

Also

$$
\psi(n)>H(n)-\delta \text {. }
$$

$$
\bar{h}(x, n) \leqq h\left(x, n, m_{n}\right) .
$$

where $n \leqq \nu(x) \leqq m_{n}$ and $\lim _{n \rightarrow \infty} \nu(x)=\infty$.

Now $\vec{h}(x, n)$ for every fixed $x$ in $I$ is a monotonically nonincreasing function by (5), and $\lim _{n \rightarrow \infty} \bar{h}(x, n)=0$ by (11) and (12). Hence we have, by Case $1, \lim _{n \rightarrow \infty} \psi(n)=0$ and $\lim _{n \rightarrow \infty} H(n)=0$, by $(10)$.

University of Texas 